\title{
Effects of radiation on mixed convection in power law fluids along a vertical wedge embedded in a saturated porous medium under prescribed surface heat flux condition
}

\author{
S. R. Qureshi, Q. Ali \& W. A. Khan \\ Department of Engineering Sciences, PN Engineering College, \\ National University of Science and Technology (NUST), Pakistan
}

\begin{abstract}
Radiation-mixed convection boundary layer analysis in power law fluids along vertical wedge embedded in a saturated porous medium has been performed using an implicit finite difference method. Darcian, steady, 2-D laminar flow has been considered under prescribed surface heat flux condition. The single mathematical model represents the case for vertical wedge, cone and plate by introducing the geometry parameter ' $\varepsilon$ '. The governing equations and boundary conditions are transformed into dimensionless form to obtain similar and non-similar solutions. The results for non-similar case have been presented and plotted. Effects of radiation parameter ' $R_{d}$ ', variable heat flux parameter ' $\lambda$ ', wedge angle parameter ' $\mathrm{m}$ ' and mixed convection parameter ' $\chi$ ' have been studied for both Newtonian and Non-Newtonian fluids. These results have also been verified with available literature in defined range of parameters and found in good agreement. Results for both Newtonian and Non-Newtonian fluids have been presented for varying parameters like local Nusselt number, temperature and velocity fields.
\end{abstract}

Keywords: vertical wedge, surface heat flu, porous medium, power law fluids.

\section{Introduction}

Heat transfer in Power Law Fluids across cylindrical surfaces has copious engineering applications. These applications comprises of areas such as underwater pollution, filtration systems, chemical, food processing, recovery of geothermal energy, crude oil extraction, pharmaceutical and thermal energy 
storage. Effects of free and mixed convection in Newtonian fluids along vertical and horizontal cylinders embedded in saturated porous medium have been studied [1-3] whereas very few analyses exist on Power law fluids along wedge [4]. Nakayama et al. [5] used Non-Darcy model to study effect of free convection over vertical flat plate and cone across saturated porous medium. Yih [6] performed natural convection effects using lateral mass flux effect on Non-Newtonian fluids along vertical cone. Mahmoud and Megahed [7] studied diffusion-thermo effects on heat and mass transfer by mixed convection of non-Newtonian power-law fluids over a vertical permeable surface embedded in a saturated porous medium. The effects of the physical parameters on the fluid temperature and concentration along with the local Nusselt number and the local Sherwood number are analyzed.

The present study is an analytical investigation concerning radiation effects on mixed convection in Power Law Fluids along vertical wedge under prescribed surface heat flux condition and embedded in a saturated porous medium. Both similar and non similar solutions have been obtained and results for non similar case have been presented. Effects of various parameters for both Newtonian and non Newtonian fluids have been reported. The results are also compared with the available data for the analysis of heat transfer in the prescribed range of parameters.

\section{Analysis}

A two dimensional, steady, laminar, and power law fluids along an impermeable vertical wedge (with half angle $\gamma$ ) embedded in a saturated porous medium under radiation-mixed convection conditions is considered for this study. Free stream velocity ' $U_{\infty}$ ' is orientated in the upward direction. The wedge surface is maintained at variable heat flux ' $q_{w}(x)$ ', which varies as $a x^{\lambda}$, where ' $\mathrm{a}$ ' is specified constant in porous medium and ' $\lambda$ ' represents the variation of heat flux along the length ' $\mathrm{x}$ ' of the wedge. Acceleration due to gravity ' $g_{x}=g \operatorname{Cos} \gamma$ ' is opposite to free stream velocity and acts vertically downward. $T_{\infty}$ represents the ambient temperature. Potential flow velocity outside the boundary layer is defined as $U_{\infty}(x)=c x^{m}$ where ' $c$ ' is prescribed constant and ' $\mathrm{m}$ ' is the wedge angle parameter. Beside viscous forces, temperature difference between wedge surface and free stream velocity will also result in additional buoyancy force. Evaluation of convective heat transfer phenomenon is of considerable importance in many industrial applications.

Considering effects of viscous dissipation and radiative heat flux ' $q_{r}$ ' to be negligible across flow direction, the flow over vertical wedge is governed by the following equations:

$$
\begin{gathered}
\frac{\partial\left(r^{\varepsilon} u\right)}{\partial x}+\frac{\partial\left(r^{\varepsilon} v\right)}{\partial y}=0 \\
u^{n}=U_{\infty}^{n}+\frac{K}{\mu} \rho g_{x} \beta\left(T-T_{\infty}\right)
\end{gathered}
$$




$$
u \frac{\partial T}{\partial x}+v \frac{\partial T}{\partial y}=\alpha\left(\frac{\partial^{2} T}{\partial y^{2}}-\frac{1}{k} \frac{\partial q_{r}}{\partial y}\right)
$$

where term ' $\varepsilon$ ' is geometry parameter. For wedge ' $\varepsilon=0$ ', for cone ' $\varepsilon=1$ ' and for plate ' $\varepsilon=m=0$ '. Equations (1)-(3) are subject to the following boundary conditions:

$$
\begin{gathered}
y=0: \quad v=0, \quad q_{w}(x)=-k\left(\frac{\partial T}{\partial y}\right)_{y=0}=a x^{\lambda} \\
y \rightarrow \infty: \quad u=U_{\infty}, \quad T=T_{\infty}
\end{gathered}
$$

Using Rosseland approximation:

$$
q_{r}=-\frac{4 \sigma}{3 \beta_{R}} \frac{\partial T^{4}}{\partial y}
$$

where $\sigma$ is the Stefan-Boltzmann constant and $\beta_{R}$ is the mean absorption coefficient. Considering temperature differences within the flow to be sufficiently small, $T^{4}$ may be defined as a linear function of temperature and expressed using Taylor series expansion as:

$$
T^{4} \approx 4 T_{\infty}^{3} T-3 T_{\infty}^{4}
$$

Invoking the following dimensionless transformations

$$
\begin{gathered}
\eta=\frac{y}{x}\left(P e_{x}^{1 / 2}+R a_{x}^{1 / 3}\right), \\
\psi=\alpha r^{\varepsilon}\left(P e_{x}^{1 / 2}+R a_{x}^{1 / 3}\right) f(\chi, \eta) \\
\theta(\chi, \eta)=\frac{\left(T-T_{\infty}\right)\left(P e_{x}^{1 / 2}+R a_{x}^{1 / 3}\right)}{\frac{q_{w}(x) x}{k}}
\end{gathered}
$$

with the dimensionless parameters defined as

$$
\begin{aligned}
\chi & =\frac{1}{1+\Omega_{x}}=\frac{P e_{x}^{1 / 2}}{P e_{x}^{1 / 2}+R a_{x}^{1 / 3}} & R_{d}=\frac{4 \sigma T_{\infty}^{3}}{k \beta_{R}} \\
\Omega_{x}=\frac{R a_{x}^{1 / 3}}{P e_{x}^{1 / 2}}, & U_{\infty}(x)=c x^{m}, & m=\frac{\gamma}{\pi-\gamma}
\end{aligned}
$$

$P e_{x}$ and $R a_{x}$ are defined as:

$$
P e_{x}=\frac{U_{\infty}(x) x}{\alpha} \quad \text { and } \quad R a_{x}=\left(\frac{x}{\alpha}\right)^{\frac{3 n}{2 n+1}}\left(\frac{\rho g_{x} \beta K q_{w}(x) x}{\mu k}\right)^{\frac{3}{2 n+1}}
$$

The governing equations (2) and (3) reduce to the following dimensionless momentum and energy equations respectively for vertical wedge, cone and plate:

$$
\left(f^{\prime}\right)^{n}=\chi^{2 n}+(1-\chi)^{2 n+1} \theta
$$




$$
\begin{aligned}
& \left(1+\frac{4 R_{d}}{3}\right) \theta^{\prime \prime}+\frac{\theta^{\prime} f}{2(2 n+1)}\{2 \varepsilon(2 n+1)+2(n+\lambda+1)+\chi(2 n m+m-2 \lambda-1)\}-\frac{\theta f^{\prime}}{2(2 n+1)} \\
& \{2 n(2 \lambda+1)+\chi(2 \lambda+1-2 n m-m)\}=\left(\frac{2 n m+m-1-2 \lambda}{2(2 n+1)}\right) \chi(1-\chi)\left\{f^{\prime} \frac{\partial \theta}{\partial \chi}-\theta^{\prime} \frac{\partial f}{\partial \chi}\right\}
\end{aligned}
$$

The term ' $\varepsilon$ ' is geometry parameter and for wedge ' $\varepsilon=0$ '.

The equations (9) and (10) for the case of wedge reduce to:

$$
\begin{gathered}
\left(f^{\prime}\right)^{n}=\chi^{2 n}+(1-\chi)^{2 n+1} \theta \\
\left(1+\frac{4 R_{d}}{3}\right) \theta^{\prime \prime}+\frac{\theta^{\prime} f}{2(2 n+1)}\{2(n+\lambda+1)+\chi(2 n m+m-2 \lambda-1)\}-\frac{\theta f^{\prime}}{2(2 n+1)} \\
\{2 n(2 \lambda+1)+\chi(2 \lambda+1-2 n m-m)\}=\left(\frac{2 n m+m-1-2 \lambda}{2(2 n+1)}\right) \chi(1-\chi)\left\{f^{\prime} \frac{\partial \theta}{\partial \chi}-\theta^{\prime} \frac{\partial f}{\partial \chi}\right\}
\end{gathered}
$$

with following transformed boundary conditions:

$$
\begin{array}{lrl}
f(0)=0 & & \theta^{\prime}(0)=-1 \\
f^{\prime}(\infty)=\chi^{2}, & \theta(\infty)=0
\end{array}
$$

where $f^{\prime}=\frac{\partial f}{\partial \eta}$ and $\theta^{\prime}=\frac{\partial \theta}{\partial \eta}$.

The local dimensionless heat transfer rate is given as:

$$
\frac{N u_{x}}{\left(P e_{x}^{1 / 2}+R a_{x}^{1 / 3}\right)}=\frac{1}{\theta(\chi, 0)}
$$

The buoyancy parameter ' $\Omega_{x}$ ' varies from zero to infinity; the case of $\Omega_{x}=0$ $\left(P_{e} \gg R_{a}\right)$ corresponds to pure forced convection $\left(U_{\infty} \neq 0, \chi=1\right)$ and $\Omega_{x}=\infty$ $\left(R_{a}>>P_{e}\right)$ corresponds to pure free convection $\left(U_{\infty}=0, \chi=0\right)$. The mixed convection parameter $\chi$ varies from 0 to 1 indicating pure free and pure forced convection, respectively. The values between $0-1$ correspond to mixed convection case.

The term ' $R_{d}$ ' is called radiation parameter and represents the effects of radiation i.e. $R_{d}=0$, it means that radiation effects are not present (Pure mixed convection case) whereas increasing values of ' $R_{d}$ ' indicate the domination of radiative heat transfer over mixed convection. The term ' $\lambda$ ' is exponent of heat flux, i.e. $\lambda=0$ represents constant heat flux case, when $\lambda>0$, the heat flux profile increases along the wedge and when $\lambda<0$, the heat flux profile decreases along length. The term ' $\mathrm{m}$ ' is wedge angle parameter and is dependent upon wedge half angle $\gamma$ (due to axi-symmetric body). 


\section{Vertical flat plate}

By setting the terms ' $\varepsilon=m=0$ ' in equations (9) and (10), following dimensionless momentum and energy equations are obtained and corresponds to the case of vertical flat plate in power law fluids under prescribed surface heat flux conditions:

$$
\begin{gathered}
\left(f^{\prime}\right)^{n}=\chi^{2 n}+(1-\chi)^{2 n+1} \theta \\
\left(1+\frac{4 R_{d}}{3}\right) \theta^{\prime \prime}+\frac{\theta^{\prime} f}{2(2 n+1)}\{2(n+\lambda+1)-\chi(2 \lambda+1)\}-\frac{\theta f^{\prime}(2 \lambda+1)}{2(2 n+1)}(2 n+\chi) \\
=\frac{\chi(2 \lambda+1)(1-\chi)}{2(2 n+1)}\left\{\theta^{\prime} \frac{\partial f}{\partial \chi}-f^{\prime} \frac{\partial \theta}{\partial \chi}\right\}
\end{gathered}
$$

By setting $R_{d}=0$ and $\mathrm{n}=1$, equations (22) and (23) represents the case for mixed convection in Newtonian fluids along vertical flat plate with variable heat flux and is subject to same boundary conditions given in equations (13) and (14):

$$
\begin{gathered}
f^{\prime}=\chi^{2}+(1-\chi)^{3} \theta \\
\theta^{\prime \prime}+\frac{\theta^{\prime} f}{6}\{2(2+\lambda)-\chi(2 \lambda+1)\}-\frac{\theta f^{\prime}(2+\chi)(2 \lambda+1)}{6}=\frac{\chi(2 \lambda+1)(1-\chi)}{6}\left\{\theta^{\prime} \frac{\partial f}{\partial \chi}-f^{\prime} \frac{\partial \theta}{\partial \chi}\right\}
\end{gathered}
$$

Values of $\theta(\chi, 0)$ for various values of mixed convection and heat flux parameters are appended in Table 3 and compared with those obtained by I Pop [8].

\section{Similar and non similar solution}

For the similar solution (first level of truncation), first derivative w.r.t ' $\chi$ ' in equations (9) and (10) are neglected. Thus the governing equations for the similar solution are:

$$
\begin{gathered}
\left(f^{\prime}\right)^{n}=\chi^{2 n}+(1-\chi)^{2 n+1} \theta \\
\left(1+\frac{4 R_{d}}{3}\right) \theta^{\prime \prime}+\frac{\theta^{\prime} f}{2(2 n+1)}\{2 \varepsilon(2 n+1)+2(n+\lambda+1)+\chi(2 n m+m-2 \lambda-1)\}- \\
\frac{\theta f^{\prime}}{2(2 n+1)}\{2 n(2 \lambda+1)+\chi(2 \lambda+1-2 n m-m)\}=0
\end{gathered}
$$

Equations (26) and (27) are subject to the boundary conditions given in equations (13) and (14). For non similar solution (second level of truncation), two 
additional variables $F(\chi, \eta)$ and $G(\chi, \eta)$ defined by $F=\frac{\partial f}{\partial \chi}$ and $G=\frac{\partial \theta}{\partial \chi}$ are introduced. Equations (9) and (10) in terms of these variables are:

$$
\begin{gathered}
\left(f^{\prime}\right)^{n}=\chi^{2 n}+(1-\chi)^{2 n+1} \theta \\
\left(1+\frac{4 R_{d}}{3}\right) \theta^{\prime \prime}+\frac{\theta^{\prime} f}{2(2 n+1)}\{2 \varepsilon(2 n+1)+2(n+\lambda+1)+\chi(2 n m+m-2 \lambda-1)\}-\frac{\theta f^{\prime}}{2(2 n+1)} \\
\{2 n(2 \lambda+1)+\chi(2 \lambda+1-2 n m-m)\}=\left\{\frac{2 n m+m-1-2 \lambda}{2(2 n+1)}\right\} \chi(1-\chi)\left(G f^{\prime}-F \theta^{\prime}\right)
\end{gathered}
$$

Two additional equations for $F$ and $G$ are obtained by differentiating equations (28) and (29) with respect to $\chi$ and by setting $\frac{\partial F}{\partial \chi}=\frac{\partial G}{\partial \chi}=0$, to give:

$$
\begin{gathered}
n\left(f^{\prime}\right)^{n-1} F^{\prime}=2 n(\chi)^{2 n-1}+\left\{G(1-\chi)^{2 n+1}-(2 n+1) \theta(1-\chi)^{2 n}\right\} \\
\left(1+\frac{4 R_{d}}{3}\right) G^{\prime \prime}+\frac{\varepsilon(2 n+1)+(n+\lambda+1)}{(2 n+1)}\left(F \theta^{\prime}+f G^{\prime}\right)+\frac{(2 n m+m-2 \lambda-1)}{2(2 n+1)} \\
\left\{\chi\left(F \theta^{\prime}+f G^{\prime}\right)+f \theta^{\prime}\right\}-\frac{n(2 \lambda+1)\left(\theta F^{\prime}+G f^{\prime}\right)}{(2 n+1)}+\frac{(2 n m+m-2 \lambda-1)}{2(2 n+1)}\left\{\chi\left(\theta F^{\prime}+G f^{\prime}\right)+\theta f^{\prime}\right\}= \\
\left\{\frac{2 n m+m-1-2 \lambda}{2(2 n+1)}\right\}\left\{\chi(1-\chi)\left(G F^{\prime}-F G^{\prime}\right)+(1-2 \chi)\left(G f^{\prime}-F \theta^{\prime}\right)\right\}
\end{gathered}
$$

Table 1: Effect of $\lambda$ on $\theta(\chi, 0)$ at $R_{d}=0$ and $\mathrm{n}=1$.

\begin{tabular}{|c|c|c|c|c|c|c|}
\hline \multirow{2}{*}{$\chi$} & \multicolumn{2}{|c|}{$\lambda=0.4$} & \multicolumn{2}{c|}{$\lambda=0$} & \multicolumn{2}{c|}{} \\
\cline { 2 - 7 } & Pop & Present & Pop & Present & Pop & Present \\
\hline 0 & 1.1403 & 1.14025 & 1.2962 & 1.2961 & 1.5914 & 1.5907 \\
\hline 0.05 & 1.1986 & 1.1961 & 1.3627 & 1.361 & 1.6734 & 1.672 \\
\hline 0.117 & 1.2795 & 1.27457 & 1.4563 & 1.45126 & 1.7911 & 1.7882 \\
\hline 0.207 & 1.3877 & 1.38127 & 1.5852 & 1.578 & 1.9592 & 1.95274 \\
\hline 0.328 & 1.5073 & 1.50594 & 1.7404 & 1.736 & 2.1835 & 2.17545 \\
\hline 0.49 & 1.5367 & 1.54906 & 1.8183 & 1.8 & 2.3723 & 2.36851 \\
\hline 0.708 & 1.2778 & 1.262 & 1.5597 & 1.5436 & 2.142 & 2.14549 \\
\hline 1 & 0.9227 & 0.92254 & 1.1262 & 1.12838 & 1.5647 & 1.56534 \\
\hline
\end{tabular}


Equations (28)-(31) are the governing equations involving four unknown functions $\mathrm{f}, \theta, \mathrm{F}$ and $\mathrm{G}$, which are to be solved subject to the boundary conditions in equation (13) and (14) and the following additional boundary conditions:

$$
F(0)=0, G^{\prime}(0)=0, \quad F^{\prime}(\infty)=\frac{2(\chi)^{2 n-1}}{\left(f^{\prime}(\infty)\right)^{n-1}} \text { and } G(\infty)=0
$$

\section{Results and discussion}

By setting ' $\varepsilon=0$ ' for wedge, the equations (28)-(31) together with boundary conditions in equations (13), (14) and (32) have been solved numerically and results are presented for varying effects of the exponent of $\operatorname{VHF}(\lambda)$, wedge angle, mixed convection parameter $(\chi)$, radiation parameter $\left(R_{d}\right)$ and power law fluids index (n). Figures 1, 2 and 3 display the variation of dimensionless local Nusselt number with varying effects of radiation parameter $\left(R_{d}\right)$, surface flux exponent $(\lambda)$ and wedge angle parameter $(\mathrm{m})$ respectively. High Nusselt number is observed across pseudo- plastic fluids $(\mathrm{n}<1)$ than Newtonian $(\mathrm{n}=1)$ and dilatant fluids $(\mathrm{n}>1)$, respectively. It is worth noting that as the mixed convection parameter increases $\chi>0.5$, the Nusselt number curves start converging and all the fluids tend to behave in a similar manner when $\chi=1$, i.e. the heat transfer phenomenon becomes independent of type of fluid at $\chi=1$. It can also be noted that for all cases, the dimensionless Nusselt initially decreases with increase of mixed convection parameter $(\chi)$, reaches to a minimum value nearly $\chi=0.5$ and then again increases gradually as $\chi>0.5$. This is due to the nature of the compound term at y-axis vs. $\chi$ plot and does not imply that the actual Nusselt number for the mixed convection is smaller than that for pure forced or pure free convection. This phenomenon is well explained by Hsieh et al. [9] for the problem of a vertical flat plate and Yih [10] for the case of vertical isothermal cone. It is also observed that wedge angle parameter has no significant effect on the Nusselt number.

Figures 4, 5 and 6 represent the effects of radiation $\left(R_{d}\right)$, surface heat flux exponent $(\lambda)$ and wedge angle on dimensionless temperature across power law fluids. It can be seen that as radiation increases, the dimensionless temperature increases for all types of fluids. Moreover, high temperature is observed across dilatant fluids $(\mathrm{n}>1)$ than Newtonian fluids $(\mathrm{n}=1)$ and pseudo plastic fluids $(\mathrm{n}<1)$, respectively. It can be seen that as $\eta$ increases, the slope of ' $\theta$ ' decreases for all curves and ultimately falls to zero as we move towards the boundary layer edges (i.e. $\eta \rightarrow \infty$ ) which confirms to the boundary condition given in equation (14) i.e. $\theta(\infty)=0$. 
It may also be noted that as surface flux exponent increases, the temperature profile decreases. Moreover, wedge angle parameter has no significance effect on temperature profile.

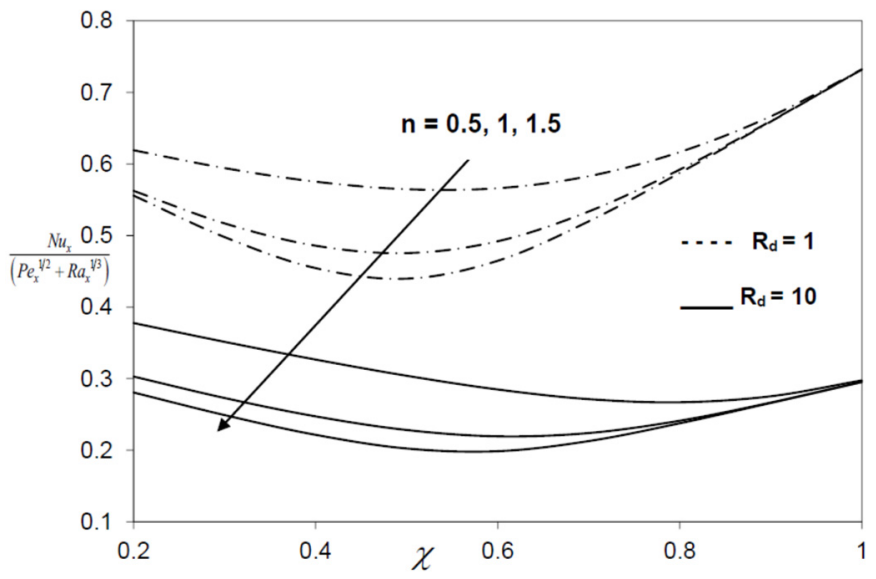

Figure 1: $\quad$ Effects of $R_{d}$ on $N u_{x}$ at $\lambda=0.5$ and $\gamma=30^{\circ}$.

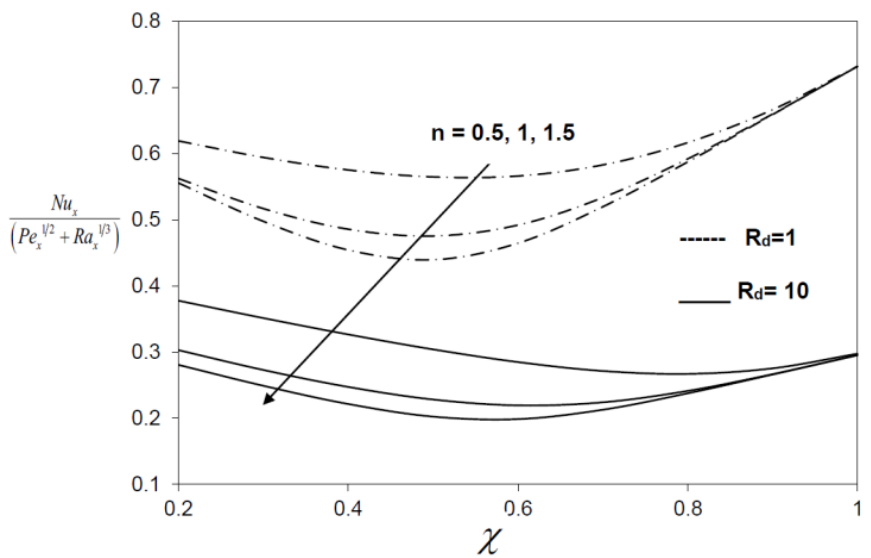

Figure 2: Effects of flux variation on $\mathrm{Nu}_{\mathrm{x}}$ at $\gamma=30^{\circ}$ and $\mathrm{R}_{\mathrm{d}}=1$. 


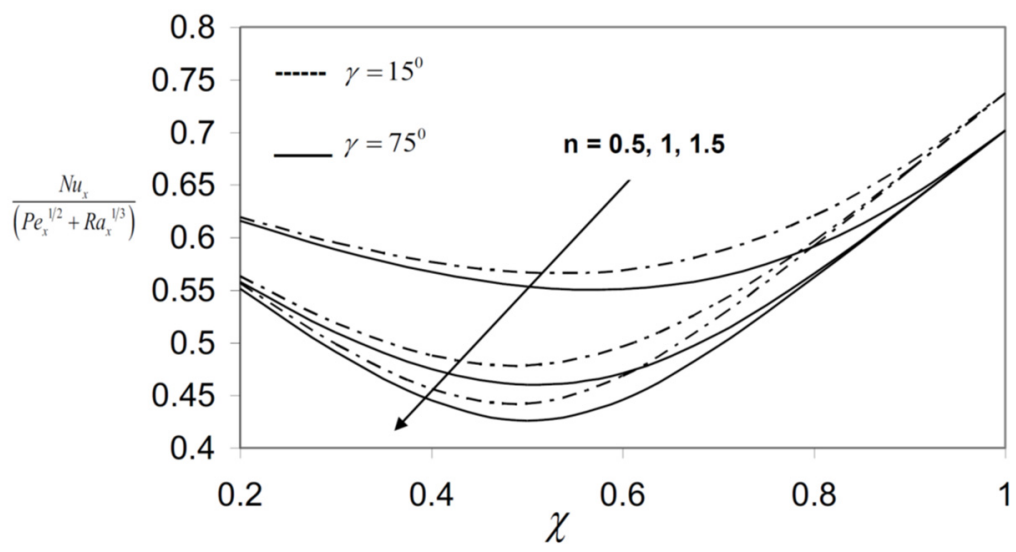

Figure 3: $\quad$ Effects of wedge angle $(\gamma)$ on $\mathrm{Nu}_{\mathrm{x}}$ at $\lambda=0.5$ and $\mathrm{R}_{\mathrm{d}}=1$.

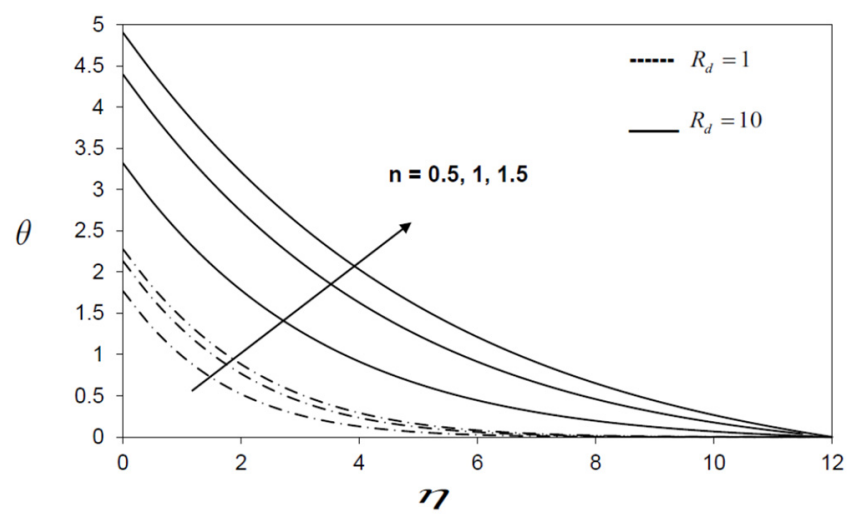

Figure 4: Radiation effects on $\theta(0.5, \eta)$ at $\lambda=0.5, \chi=0.5$ and $\gamma=30^{\circ}$.

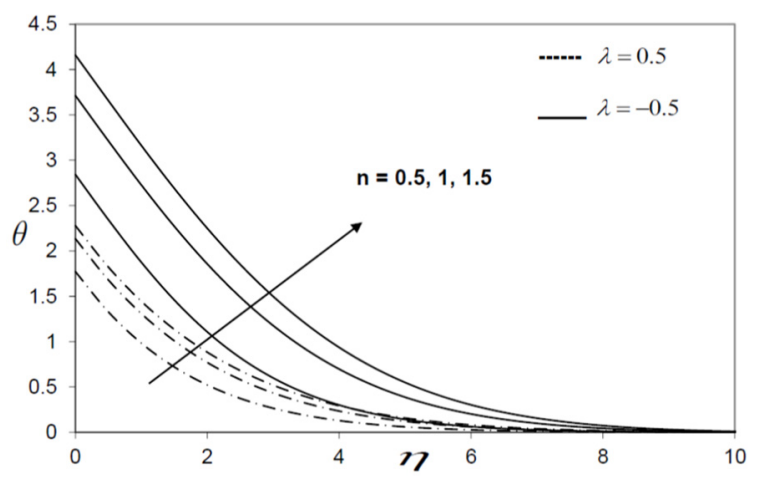

Figure 5: Effects of $\lambda$ on $\theta(0.5, \eta)$ at $\mathrm{R}_{\mathrm{d}}=1$ and $\gamma=30^{\circ}$. 


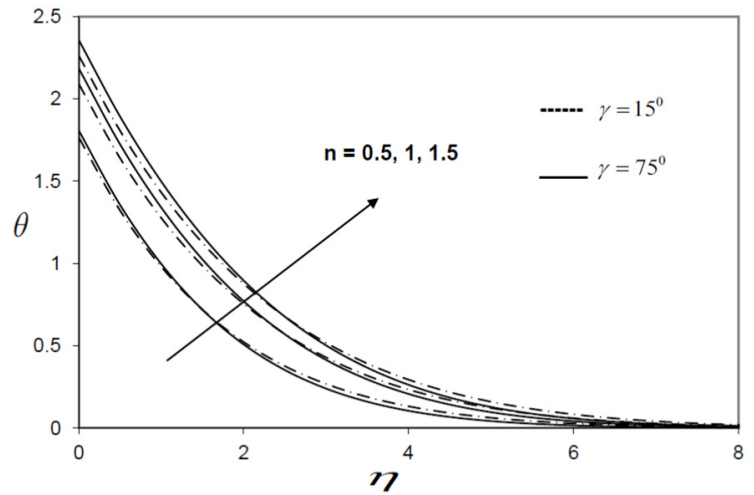

Figure 6: Effects of wedge angle on $\theta(0.5, \eta)$ at $\mathrm{R}_{\mathrm{d}}=1$ and $\lambda=0.5$.

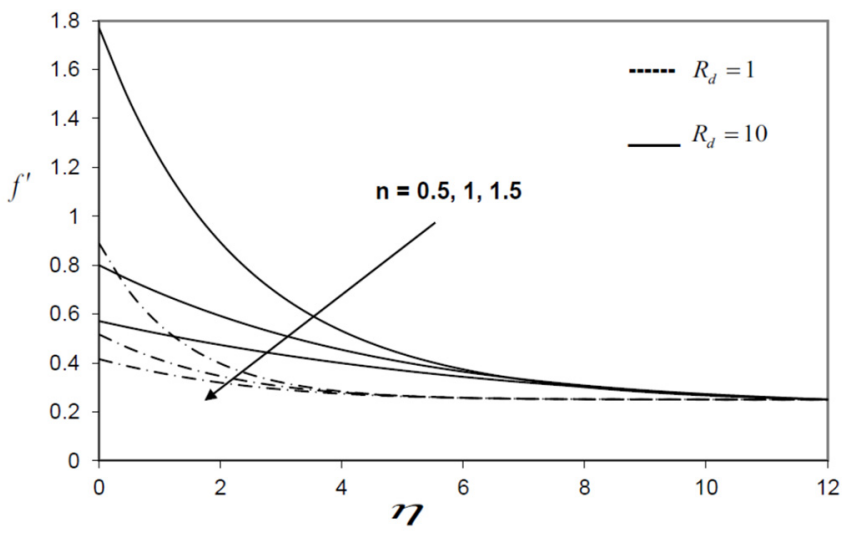

Figure 7: Radiation effects on $f^{\prime}(0.5, \eta)$ at $\lambda=0.5$ and $\gamma=30^{\circ}$.

Figures 7, 8 and 9 represent the effects of radiation, surface flux exponent and wedge angle parameter on dimensionless velocity profile for power law fluids. It can be seen that irrespective of the type of the fluid, as ' $\eta$ ' increases, the slope of the velocity tends to decreases and velocity becomes constant at higher values of ' $\eta$ ' (i.e. we move towards the boundary layer edges). Moreover, high velocity is observed across pseudo plastic fluids $(\mathrm{n}<1)$ than Newtonian $(\mathrm{n}=1)$ and dilatant fluids $(n>1)$, respectively. It is due to the fact that as viscosity increases, the skin friction co-efficient increases which tend to retard the velocity. It is also observed that wedge angle parameter has no significant effect on flow velocity. 


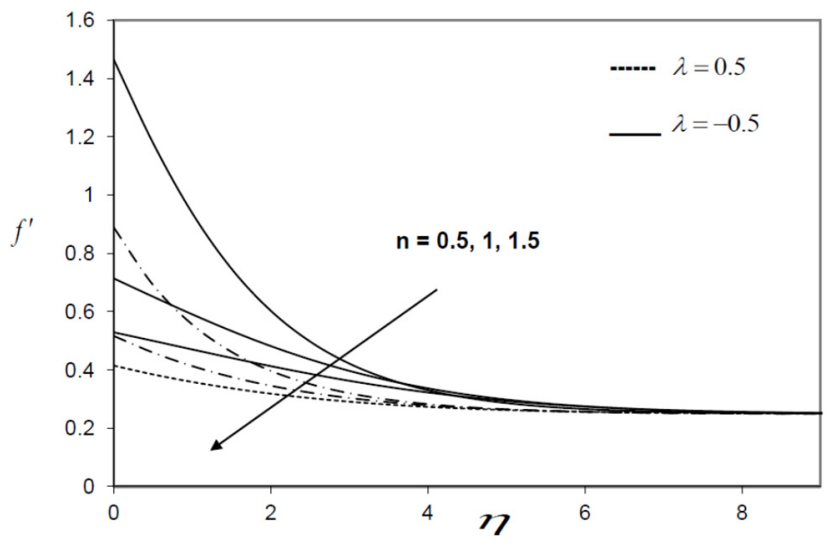

Figure 8: Effects of flux variation on $f^{\prime}(0.5, \eta)$ at $\mathrm{Rd}=1$ and $\gamma=30^{\circ}$.

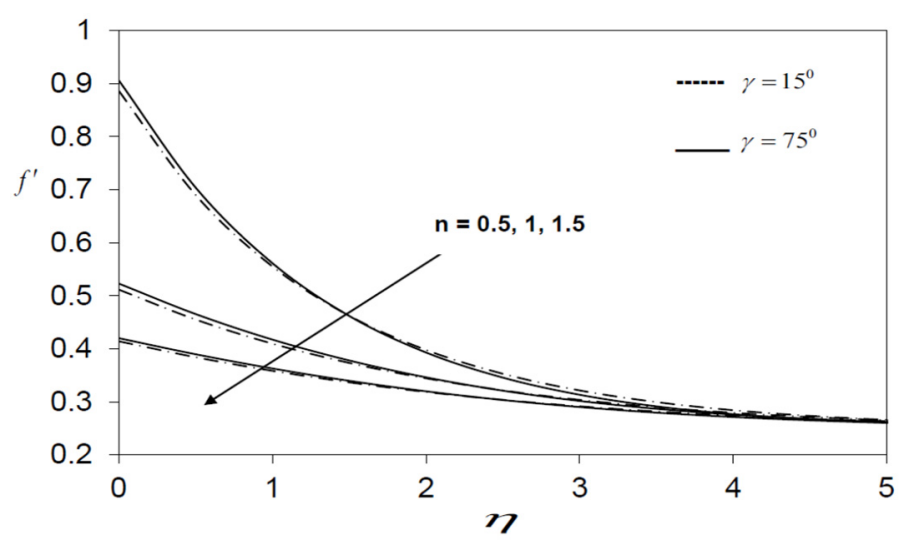

Figure 9: Effects of wedge angle on $f^{\prime}(0.5, \eta)$ at $\mathrm{R}_{\mathrm{d}}=1$ and $\lambda=0.5$.

\section{Conclusion}

The heat transfer in radiation-mixed convection about vertical wedge embedded in a saturated porous medium has been analyzed for the case of prescribed surface heat flux. Effects of various parameters on dimensionless local Nusselt number, temperature and velocity fields have been presented. It is found that as the radiation parameter or wedge angle is increased, the Nusselt number decreases whereas it increases with increase in the value of $\lambda$ at a given value of $\chi$. Also, it is observed that as viscosity increases, the skin friction co-efficient increases which tend to reduce the velocity. Moreover, pseudoplastic fluids are more heat conductive than Newtonian and dilatant fluids respectively and all fluids behave identically at $\chi=1$. 


\section{References}

[1] Merkin J.H, Free convection boundary layer on an isothermal horizontal cylinder, ASME AIChE Heat Transfer Conference 76-HT-16, 1-4, 1976.

[2] Ingham D.B, The Non-Darcy free convective boundary layer on axisymmetric and two dimensional bodies of arbitrary shape, Int. J. Heat Mass transfer Vol. 29, pp. 1759-1760,1986.

[3] I. Pop, D.B Ingham \& P Cheng, Transient free convection about a horizontal circular cylinder in a porous medium, Fluid Dynamics Research Vol. 12, pp. 295-305, 1993.

[4] Waqar A. Khan \& Rama Subba Reddy Gorla, Mixed convection of powerlaw fluids along a vertical wedge with convective boundary condition in a porous medium, Journal of Mechanical Science and Technology 24 (9), pp. 1919-1925, 2010.

[5] A. Nakayama, T. Kokudai and H. Koyama, An integral treatment for NonDarcy free convection over a vertical flat plate and cone embedded in a fluid saturated porous medium, Warme-und Stoffubertragung Vol. 23, pp. 337341, 1988.

[6] K.A. Yih, Uniform lateral mass flux effect on natural convection of NonNewtonian fluids over a cone in a porous medium, Int. Comm. Heat Mass Transfer, Vol. 25, No. 7, pp. 959-968, 1998.

[7] Mahmoud, M., Megahed, A. Thermal radiation effect on mixed convection heat and mass transfer of a non-Newtonian fluid over a vertical surface embedded in a porous medium in the presence of thermal diffusion and diffusion-thermo effects, Journal of Applied Mechanics \& Technical Physics; Jan 2013, Vol. 54 Issue 1, p. 90.

[8] Ioan Pop, Tsung-Yen Na, Darcian mixed convection along slender vertical cylinder with variable surface heat flux embedded in a porous medium", Int. Comm. Heat Mass Transfer, Vol. 25, No. 2, pp. 251-260, 1998.

[9] J.C. Hsieh, T.S. Chen \& B.F. Armaly, Mixed convection along a non isothermal vertical flat plate embedded in a porous medium: the entire regime, Int. Journal Heat Mass Transfer, Vol. 36, No. 7, pp. 1819-1825, 1993.

[10] K.A. Yih, Radiation effect on mixed convection over an isothermal cone in a porous medium, Int. Comm. Heat Mass Transfer, Vol. 37, pp. 3-57, 2001. 